\title{
Estratégias de gestão de carteiras de investimentos
}

\author{
João Luis Gonçalves " \\ DAMAT/UTFPR, Curitiba, PR \\ Ronie Perterson Darid" \\ DAMAT/UTFPR, Curitiba, PR \\ Patrik Borges de Miranda \\ UTFPR, Curitiba, PR
}

Resumo. Analisamos o desempenho de estratégias de investimento baseadas no clássico método de Média Variância de Markowitz e em uma de suas variações, o método de Média Variância Penalizada, na gestão de uma carteira de investimentos em ações brasileiras. A estratégia baseada no Média Variância Penalizada permite maior controle na relação risco×retorno do que a estratégia baseada no Média Variância de Markowitz. Comparamos numericamente o desempenho quanto a risco e retorno destas estratégias com outras que não se utilizam de métodos de otimização.

Palavras-chave: alocação de carteiras, métodos de média variância, equal weighting.

\section{Introdução}

Em reunião no início de agosto de 2020, o Comitê de Política Monetária (COPOM) reduziu a taxa básica de juros da economia brasileira - taxa SELIC - para $2 \%$ ao ano, a mínima histórica. Se considerarmos a meta de inflação de $2020 \mathrm{em} 4 \%$ ao ano, a taxa de juros real brasileira estava negativa em $2 \%$ ao ano. A SELIC nesse nível implica que a poupança rende apenas $1.4 \%$ ao ano, fazendo com que os brasileiros que investem na poupança percam poder de compra. Essa conjuntura explica em parte a migração de investidores da renda fixa para investimentos de risco, especialmente o investimento em ações. De fato, o número de investidores na Bolsa de Valores $\left(\mathrm{B}^{3}\right)$ atingiu três milhões e um crescimento de mais de $80 \%$ em 2020.

É razoável supor que esse contingente de novos investidores é composto principalmente por pessoas que não estão em busca de lucros rápidos, operando com opções ou com day-trade. Estudo recente [3] indica que menos de $1 \%$ das pessoas que se dispõe ao day-trade tem sucesso após um ano no mercado. Desta forma, a análise de estratégias de investimentos de médio e longo prazo ganha ainda mais importância no momento atual, sejam elas focadas em redução de risco e/ou busca de maior rentabilidade.

A Teoria Moderna das Carteiras admite risco de um ativo como a variância dos seus retornos e o risco de uma carteira depende da covariância entre os retornos dos ativos que a compõe. Estratégias para a redução de risco de investimento em crises no mercado brasileiro foram estudadas em [2], no caso da crise de 2008, e em 7, na crise provocada pela pandemia de COVID-19.

Neste trabalho, baseado em [10], analisamos o desempenho de estratégias de gestão de uma carteira de investimentos formada por doze ações (Tabela 1).

\footnotetext{
1jlgoncalves@utfpr.edu.br

2 ronie@utfpr.edu.br

${ }^{3}$ patrikborges@hotmail.com
} 
Essas estratégias buscam determinado nível de retorno, com o menor risco possível, e utilizam o clássico método de Média Variância de Markowitz (MVM), e também uma de suas variações, o método de Média Variância Penalizada (MVP). O período de análise foi de janeiro de 2019 a agosto de 2020, e compreende parte da crise econômica causada pela COVID-19.

A grande dificuldade dos métodos de média variância está na estimativa dos retornos esperados. Percebemos neste trabalho que o ambiente de crise torna essa estimação ainda mais complicada.

Comparamos os resultados obtidos com três estratégicas básicas de gestão de carteiras: a distribuição igualitária do capital na carteira (3), a estratégia Buy and Hold (5), e o tradicional método 60/40 (4), no qual utilizamos como ativo livre de risco o título do Tesouro Direto conhecido como Tesouro SELIC (antiga LFT), com vencimento em 01 de março de 2021.

\section{Métodos de gestão de carteiras de investimento}

Uma carteira de investimentos é um composta por um conjunto de ativos numerados de 1 até $n$ e seus respectivos pesos. Assim, uma carteira $x$ pode ser entendida como um vetor

$$
x(t)=\left(x_{1}(t), \ldots, x_{n}(t)\right), \text { tal que } x_{1}(t)+\ldots+x_{n}(t)=1,
$$

onde $x_{i}(t)$ é o peso do ativo $i$ no tempo $t$. O peso de um ativo $i$ na carteira pode ser explicitamente definido considerando o seu preço $p_{i}(t)$, sua quantidade $\alpha_{i}(t)$ e o valor da carteira $\left(V_{x}(t)\right)$. Assim,

$$
x_{i}(t)=\frac{\alpha_{i}(t) p_{i}(t)}{V_{x}(t)} .
$$

Uma sequência de carteiras é denominada uma estratégia de investimento, sendo que cada termo da sequência é obtido pelo rebalanceamento dos pesos da carteira anterior.

Por exemplo, a estratégia Equal Weighting (EW), também chamada de $1 / n$, trata de dividir igualmente o capital total da carteira entre os $n$ ativos que a compõe, ou seja, define

$$
x_{i}=\frac{1}{n}, \text { para } i=1, \ldots, n .
$$

Já a estratégia tradicional aloca $40 \%$ do capital disponível em renda fixa, considerada livre de risco, e os $60 \%$ restantes distribuídos igualmente em ações. Assim,

$$
x_{1}=\ldots=x_{n}=\frac{0.6}{n} \text { e } x_{n+1}=0.4 .
$$

Não rebalancear a carteira e deixar os percentuais se autorregularem conforme a variação dos preços de mercado é a estratégia que chamamos de Buy and Hold (BH). Consideramos a alocação inicial conforme (3) e na sequência utilizamos (2) para atualizar os pesos por

$$
x_{i}(t+1)=\frac{\alpha_{i} p_{i}(t+1)}{V_{x}(t+1)}=\frac{\alpha_{i} p_{i}(t)\left(1+r_{i}(t+1)\right)}{(1+r(t+1)) V_{x}(t)}=\left(\frac{1+r_{i}(t+1)}{1+r(t+1)}\right) x_{i}(t),
$$

onde $r_{i}(t+1)$ é o retorno obtido pelo ativo $i$ e $r(t+1)$ o retorno obtido pela carteira $x$, de $t$ a $t+1$.

\subsection{O Método de Média Variância de Markowitz (MVM)}

A Teoria das Carteiras teve origem em 1952, com o pioneiro trabalho de Harry Markowitz 9], cuja principal contribuição foi a formalização matemática da ideia de diversificação de investimentos, mostrando que a correlação negativa entre os retornos dos diferentes ativos de risco que 
compõe uma carteira pode ser utilizada para reduzir o risco da carteira como um todo, para um dado nível de retorno desejado.

O preço (cotação de mercado) de um ativo em um dado tempo futuro é uma variável aleatória discreta, que por definição assume somente valores positivos. Dados os ativos de risco $1, \ldots, n$, o investidor tem o problema de definir os pesos $x_{1}, \ldots, x_{n}$ destes ativos na carteira de tal forma que $x_{1}+\ldots+x_{n}=1$ e $x_{i} \geq 0$, para todo $i=1, \ldots, n$. [

Fixado um ativo $i$ e um intervalo de tempo $I=[s, t]$, seja $R_{i}$ a variável aleatória (também finita e discreta) que representa o retorno de $i$ no intervalo $I$. Sejam $m_{1}, \ldots, m_{k}$ os possíveis valores para $R_{i}$ e $p_{1}\left(m_{1}\right), \ldots, p_{k}\left(m_{k}\right)$ as respectivas probabilidades destes valores ocorrerem. O retorno esperado do ativo $i$ no intervalo $I$ é denotado por $\mu_{i}=E\left(R_{i}\right)$ e é entendido como o conceito estatístico de valor esperado do retorno de $i$ em $I$. Mais precisamente, $E\left(R_{i}\right)=m_{1} p_{1}\left(m_{1}\right)+\ldots+m_{k} p_{k}\left(m_{k}\right)$.

Sendo $R=\left(R_{1}, \ldots, R_{n}\right)$ o vetor composto pelos retornos dos ativos da carteira $x=\left(x_{1}, \ldots, x_{n}\right)$, o retorno de $x$ em $I$ é $R x^{T}=R_{1} x_{1}+\ldots+R_{n} x_{n}$. Considerando a linearidade do valor esperado, podemos definir o retorno esperado da carteira $x$ no intervalo $I$ como

$$
\mu=E(R)=\sum_{j=1}^{n} x_{j} E\left(R_{j}\right)=E x^{T}, \text { onde } E=\left(E\left(R_{1}\right), \ldots, E\left(R_{n}\right)\right) .
$$

Para um ativo em particular, o risco é traduzido no contexto do trabalho de Markowitz [9] utilizando a variância dos retornos deste ativo com relação ao seu retorno esperado. Denotamos por $\sigma_{i j}=\operatorname{cov}(i, j)$ a covariância entre os ativos $i$ e $j$ da carteira $x, \sigma_{i}^{2}$ a variância do ativo $i$ e $M_{i j}=\sigma_{i j}$ a matriz de covariância. Assim, o risco da carteira $x$ é definido como o desvio padrão

$$
\sigma(x)=\left[\sum_{i=1}^{n} x_{i}^{2} \sigma_{i}^{2}+\sum_{i=1}^{n} \sum_{j \neq i}^{n} x_{j} \sigma_{i j}\right]^{1 / 2}=\left[x M x^{T}\right]^{1 / 2} .
$$

É impossível simultaneamente maximizar $\mu$ e minimizar $\sigma$. Desta forma, é assumido que o investidor racional deve escolher fixar um dos parâmetros e otimizar o outro, buscando obter o quê Markowitz chamou de uma carteira eficiente. A cada carteira corresponde um ponto no plano risco $\times$ retorno e o conjunto de todos os pontos associados a carteiras eficientes é chamado de Fronteira Eficiente. O processo de otimização que descrevemos é chamado de Método de Média Variância de Markowitz (MVM). Neste trabalho escolhemos minimizar risco para um retorno esperado fixado, resolvendo o problema de otimização quadrática convexa dado por:

$$
\min _{x \in \mathbb{R}^{n}} \frac{1}{2} x M x^{T} \text {, s.a } \mathbf{1} x^{T}=1, E x^{T}=\mu_{0} \text { e } x_{i} \geq 0, i=1, \ldots, n .
$$

onde $\mathbf{1}=(1, \ldots, 1)$ é o vetor com $n$ entradas iguais a 1.

O menor valor possível para a escolha de $\mu_{0}$ é denotado por $\mu_{\text {min }}$. Corresponde à carteira de mínima variância, obtida retirando a segunda restrição do Problema (8). Nesta versão, o problema foi estudado em 7. Por outro lado, o maior valor para $\mu$ é denotado por $\mu_{\max }$ e que corresponde ao retorno esperado do ativo com maior retorno esperado da carteira, que implicará na concentração total da carteira nesse ativo. A Fronteira Eficiente é obtida fazendo $\mu_{0}$ variar no intervalo $\left[\mu_{\min }, \mu_{\max }\right]$. Portanto, o problema do investidor racional é escolher uma dessas carteiras, dependendo de quanto risco (ou retorno) deseja assumir.

A matriz $M$ é simétrica e semidefinida positiva por ser a matriz de covariância do vetor randômico $R$. Assim, a função objetivo em (8) é quadrática e limitada inferiormente. As restrições são lineares. Portanto, a existência e unicidade de solução do Problema (8) segue de [8, Teorema 1.34], originalmente apresentado em [5] e [1].

\footnotetext{
${ }^{4}$ Admitir pesos negativos corresponderia à possibilidade de vendas a descoberto na carteira. Markowitz não considera este caso em 9]. O leitor interessado pode consultar 4 .
} 


\subsection{O Método de Média Variância Penalizada (MVP)}

Uma variação do método de Média Variância de Markowitz encontrado na literatura [11] consiste em penalizar a função objetivo de (8) usando o retorno esperado. Em contrapartida desta penalização, não há restrições quanto ao retorno, diferente do que ocorre no Problema (8). Dessa forma, a função objetivo possui um parâmetro, representado por $\alpha$, que faz a ponderação entre risco e retorno. Para uma melhor representatividade do papel de $\alpha$, normalizamos as funções risco e retorno. O novo problema é dado por

$$
\min _{x \in \mathbb{R}^{n}} \alpha \frac{x M x^{T}}{\|M\|}-(1-\alpha) \frac{E x^{T}}{\|E\|}, \text { s.a } \mathbf{1} x^{T}=1 \text { e } x_{i} \geq 0, i=1, \ldots, n .
$$

De acordo com [12], podemos encontrar as carteiras eficientes, como apresentado no Problema (8), fazendo $\alpha$ variar no intervalo [0,1]. Por exemplo, para $\alpha=1$, a solução do Problema (9) corresponde à carteira de mínima variância, que é a de menor risco e menor retorno esperado dentre as carteiras da Fronteira Eficiente. Para $\alpha=0$, a solução indica a carteira eficiente de maior retorno esperado e maior risco, pois na função objetivo resta apenas o retorno esperado $E$ com sinal negativo.

Nos casos particulares de $\mu=\mu_{\min }$ e $\mu=\mu_{\max }$, o Problema (8) é equivalente ao Problema (9) com $\alpha=0$ e $\alpha=1$, respectivamente. Apesar dessas equivalências e de ambos os problemas terem como solução carteiras eficientes, não temos uma relação explícita entre $\mu$ e $\alpha$ que torne os problemas equivalentes. Isto será comprovado nos experimentos numéricos. A existência e unicidade de solução para (9) segue o exposto para (8).

\section{Metodologia}

Os dois métodos apresentados nas seções anteriores foram aplicados semanalmente para o ajuste da carteira, definindo assim estratégias as investimento que continuamos a chamar de MVM e MVP. Também foram utilizadas as estratégias básicas EW, BH e Tradicional, conforme (3), (4) e (5).

Os problemas de otimização foram resolvidos utilizando a função quadprog do software Matlab@. em sua versão R2015a, que escolhe o método de resolução mais apropriado para cada problema.

A carteira que consideramos foi composta por 12 ações negociadas na Bolsa de Valores - $\mathrm{B}^{3}$, conforme a Tabela 1, de forma que as empresas representassem os diversos setores da economia e que constassem do índice Ibovespa em janeiro de 2019.

Tabela 1: Ativos utilizados

\begin{tabular}{c|c|c|c|c|c} 
N. & Ticker & Nome & N. & Ticker & Nome \\
\hline 1 & ABEV3 & Ambev S.A. & 7 & RADL3 & Raia Drogasil S.A. \\
\hline 2 & EQTL3 & Equatorial Energia S.A. & 8 & RENT3 & Localiza R.C. S.A. \\
\hline 3 & IGTA3 & Iguatemi E. S. S.A. & 9 & SBSP3 & SABESP S.A. \\
\hline 4 & ITUB4 & Itaú Unibanco S.A. & 10 & VALE3 & Vale S.A. \\
\hline 5 & MGLU3 & Magazine Luiza SA & 11 & VIVT4 & Telefônica Brasil S.A. \\
\hline 6 & PETR4 & PETROBRÁS S.A. & 12 & WEGE3 & WEG S.A.
\end{tabular}

Foram coletados os preços de fechamento semanais dessas ações no período de 28 de dezembro de 2017 a 28 de agosto de 2020, através dos dados disponíveis em [6]. A partir desses dados foram 
calculados os retornos históricos semanais, no total de 139 semanas. Não foram considerados ajustes por juros ou dividendos, e nem taxas de negociação ou impostos.

As matrizes de covariância foram calculadas semanalmente utilizando retornos dos ativos das 52 semanas anteriores. Da mesma forma, os retornos esperados consideram as médias dos retornos da mesmas semanas.

No caso do MVM foi estabelecido um retorno esperado para a carteira $\left(\mu_{0}\right)$ entre $1 \%$ e a média dos retornos esperados das ações no período, o que for maior. Quando esta escolha resultar em um conjunto viável vazio para o Problema (8), é aplicada uma redução no valor de $\mu_{0}$, de modo a obter solução numérica para o problema de otimização.

\section{Experimentos Numéricos}

Na estratégia MVP, não há um critério inicial para a escolha do parâmetro $\alpha$. Consideramos duas possibilidades, $\alpha=0.5$ e também $\alpha=0.95$. Na Figura 1 são apresentados os retornos acumulados pelas estratégias MVM, MVP, BH, EW, Tradicional e também pelo índice Ibovespa, a título de comparação. A estratégia de maior destaque foi a MVP $\operatorname{com} \alpha=0.5$, pois dessa forma

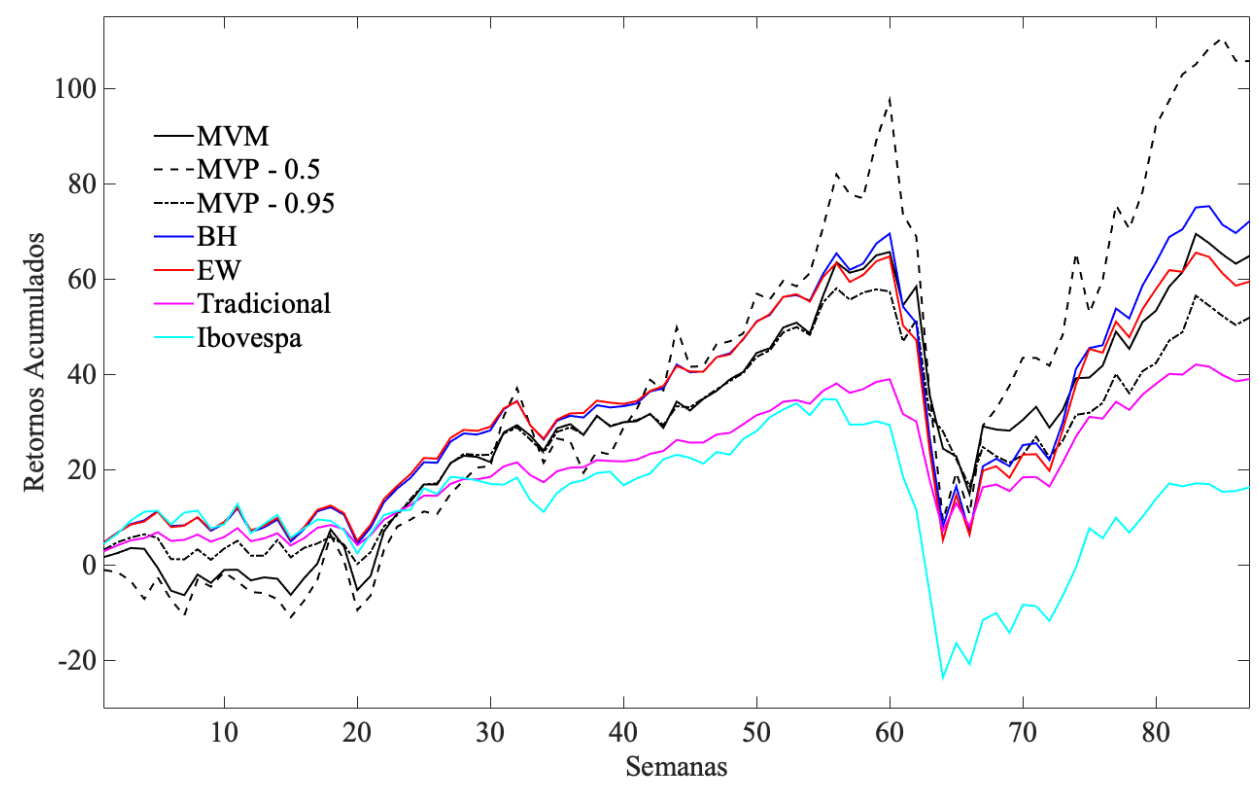

Figura 1: Retornos Acumulados

grande parte do objetivo do Problema (9) passa a ser maximizar o retorno.

As estratégias MVM, BH e EW apresentaram comportamentos parecidos, ainda que nas primeiras e nas últimas semanas as diferenças sejam significativas. A estratégia Tradicional, por sua natureza conservadora tem menores oscilações e também menores retornos. Pelo aporte em ativo livre de risco, no período da crise, ela conseguiu amenizar bastante a queda.

Na Figura 2, podemos ver o risco (em porcentagem) para as estratégias consideradas. De forma geral, os riscos apresentam comportamento bastante consistente com os retornos de cada estratégia, sendo os maiores riscos associados aos maiores retornos. 


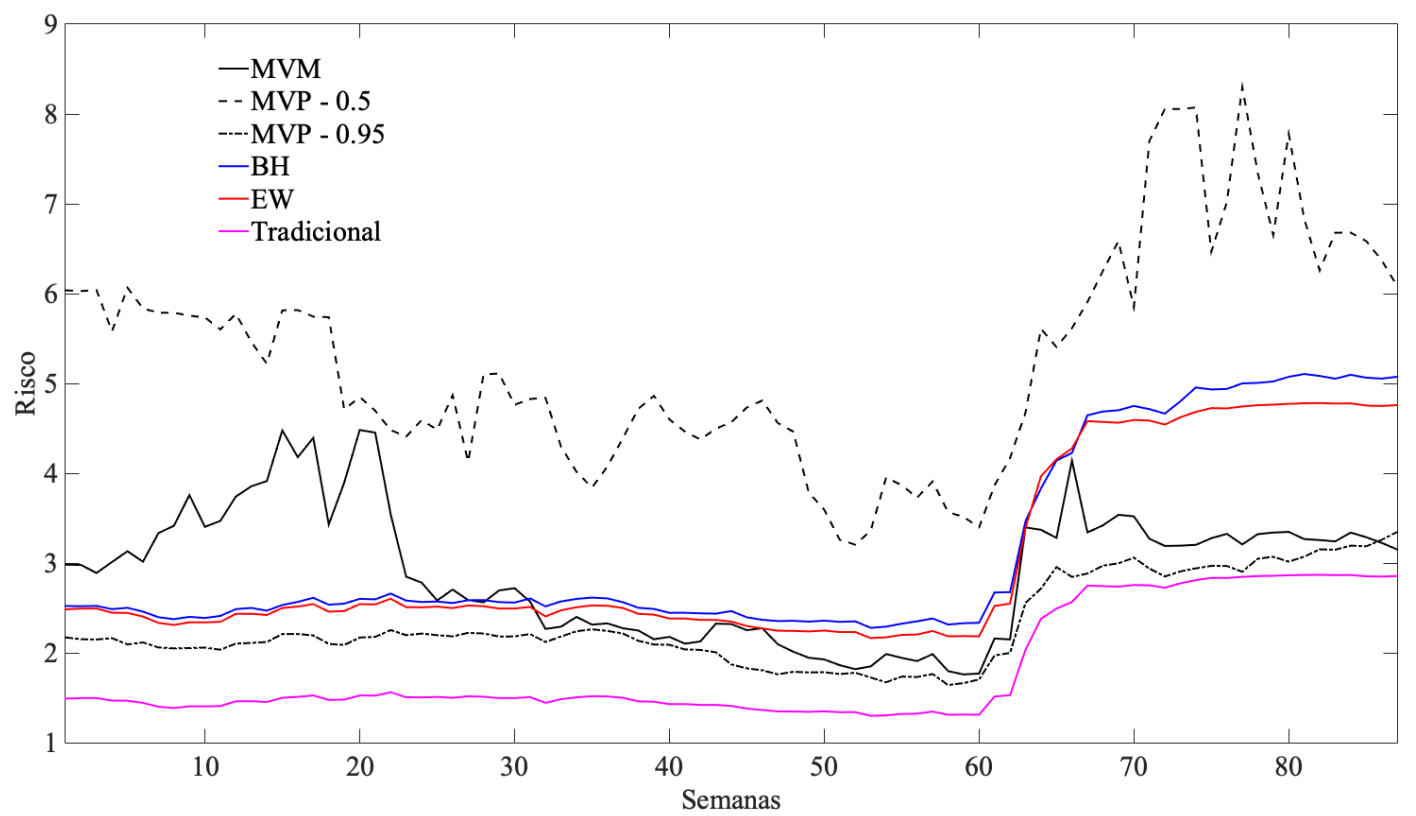

Figura 2: Riscos

Dessa análise geral destoam o MVM e o MVP $\operatorname{com} \alpha=0.5$ no período da primeira a vigésima terceira semana, em que os maiores riscos não produziram retornos superiores. Também destoa da análise geral, o MVM no período entre as semanas 67 e 87 em que, em comparação com as estratégias $\mathrm{BH}$ e EW, com menor risco tem o mesmo nível de retorno.

O parâmetro $\alpha=0.5$ torna a estratégia MVP pouco comparável com as demais, pois riscos e retornos são maiores. Além disso, o risco dessa estratégia apresenta oscilações que estão associadas a concentração dos investimentos em poucos ativos, tendo alocado mais de $90 \%$ do capital em apenas um ativo na maioria das semanas. Com $\alpha=0.95$, o MVP faz melhor jus a denominação de Penalizado, pois uma penalização de $50 \%, \alpha=0.5$, acaba por equiparar o risco e o retorno.

Podemos constatar que o comportamento do retorno acumulado do MVP com $\alpha=0.95$ oscila muito menos que com $\alpha=0.5$. No risco a diferença é ainda mais perceptível. O risco do MVP com $\alpha=0.95$ foi maior apenas que o da estratégia Tradicional e não apresentou oscilações significativas. Apesar disso, em grande parte do período, o nível de retorno está próximo das demais estratégias, sendo inferior no período posterior a crise. Parte do desempenho abaixo do esperado das estratégicas MVM e MVP deve-se à falta de precisão das estimativas para o retorno esperado.

\section{Conclusões}

Apresentamos o clássico método de Média Variância de Markowitz e uma variação na qual a restrição que impunha o retorno é substituída por uma penalização, associada ao retorno, na função objetivo. Propomos a utilização desses métodos como estratégia de investimento em uma carteira de ações, com rebalanceamento semanal. Aplicamos essas estratégias utilizando o histórico de retornos dessas ações, analisando um período que inclui uma crise, o que torna o cenário mais interessante para a análise dos métodos. 
As piores semanas da crise impactaram menos o desempenho das estratégias MVM e MVP com $\alpha=0.95$. Cabe ressaltar que a escolha do parâmetro $\alpha$ no MVP afeta risco, retorno e diversificação da carteira. Apesar da necessidade de definir o parâmetro $\alpha$, que deve variar conforme a carteira e o perfil do investidor, automaticamente o nível de retorno é otimizado para cada $\alpha$, ao passo que para o MVM impor um retorno à carteira, que mantenha o problema solúvel, é um problema a mais a ser resolvido.

Um problema de otimização mais fácil e implementação numérica mais estável são outros pontos positivos do MVP. Ainda, sob as mesmas condições que o MVM, o MVP não obteve picos tão altos de risco.

\section{Agradecimentos}

À Sociedade Brasileira de Matemática, que viabilizou a implementação do PROFMAT.

\section{Referências}

[1] Blum, E.; Oettli, W., Technical Note - Direct proof of the Existence Theorem for Quadratic Programming. Operations Research, Vol. 20, No. 1, p. 165-167, 1972.

[2] Bortoluzzo, M., et al, Comparação do desempenho de carteiras utilizando os métodos paridade de risco, mínima variância e equal weighting: um estudo no mercado brasileiro em períodos pré, durante e pós a crise de 2008. Revista Evidenciação Contábil E Finanças, Vol 6, No 3, p. 36-53, 2018.

[3] Chague, F.; Giovanetti, B. É possível viver de day-trade em ações? Brazilian Review of Finance (Online), Vol. 18, n. 3, p. 1-4, 2020.

[4] Capinski, M., Zastawniak, T., Mathematics for Finance: An Introduction to Financial Engineering. Springer, 2006.

[5] Frank, M.; Wolfe, P. An algorithm for quadratic programming. Naval Research Logistics Quarterly, Vol. 3. p. 95-110, 1956.

[6] Investing.com, Filtro de ações. Disponível em https://br.investing.com. Acesso em: 01 set. 2020.

[7] Jacob, M. L. A., Estratégias de gestão de risco de investimento no Brasil durante a pandemia de COVID-19. Dissertação de Mestrado, Universidade Tecnológica Federal do Paraná, Curitiba, 2021. Disponível em: http://repositorio.utfpr.edu.br/jspui/handle/1/24663.

[8] Krulikovski, E. H. M. Análise teórica de máquinas de vetores suporte e aplicação a classificação de caracteres. Dissertação de Mestrado, Universidade Federal do Paraná, Curitiba, 2017.

[9] Markowitz, H., Portfolio Selection The Journal of Finance, Vol. 7, No. 1, p. 77-91, 1952.

[10] Miranda, P. B., Estratégias de gestão de carteiras de investimento no mercado brasileiro. Dissertação de Mestrado, Universidade Tecnológica Federal do Paraná, Curitiba, 2021. Disponível em http://repositorio.utfpr.edu.br/jspui/handle/1/24662

[11] Nocedal, J., Wright, S. J., Numerical Optimization. Springer, 2006.

[12] Wang, S.; Xia,Y., Portfolio Selection and Asset Pricing . Springer ,2002. 